Analysis

\title{
Cooperation for sustainable forest management: An empirical differential game approach is
}

\author{
Pablo Andrés-Domenech ${ }^{\mathrm{a}, \mathrm{b}}$, Guiomar Martín-Herrán ${ }^{\mathrm{b}, \mathrm{c}}$, Georges Zaccour ${ }^{\mathrm{b}, \mathrm{d}, *}$ \\ a LEF, AgroParisTech and INRA, UMR356 Nancy, France \\ b GERAD, Canada \\ c IMUVA, Instituto de Matemáticas, Universidad de Valladolid, Spain \\ d HEC Montréal, Canada
}

\section{A R T I C L E I N F O}

\section{Article history:}

Received 22 September 2014

Received in revised form 9 June 2015

Accepted 15 June 2015

Available online $\mathrm{xxxx}$

\section{Keywords:}

Deforestation

Sustainable forest

Dynamic games

optimal control

Time consistency

Emissions

\begin{abstract}
A B S T R A C T
We model the role of the world's forests as a major carbon sink and consider the impact that forest depletion has on the accumulation of $\mathrm{CO}_{2}$ in the atmosphere. Two types of agents are considered: forest owners who exploit the forest and draw economic revenues in the form of timber and agricultural use of deforested land; and a non-forest-owner group who pollutes and suffers the negative externality of having a decreasing forest stock. We retrieve the cooperative solution for this game and show the cases in which cooperation enables a partial reduction in the negative externality. We analyze when it is jointly profitable to abate emissions, when it is profitable to reduce net deforestation, and when it is optimal to do both (abate and reduce net deforestation). Assuming that the players adopt the Nash bargaining solution to share the total dividend of cooperation, we determine the total amount that the non-forest owners have to transfer to forest owners. Next, we define a time-consistent payment schedule that allocates over time the total transfer.
\end{abstract}

(c) 2015 Elsevier B.V. All rights reserved.

\section{Introduction}

The world's forests cover nearly one-third of the Earth's surface, but are decreasing at an alarming rate, with an area equivalent to the size of Costa Rica being deforested every year (FAO, 2010). World deforestation has become an issue of great international environmental concern for a number of reasons: first, the world's forests have an ecological value as carbon sinks. Second, forests host much of the world's biodiversity. Third, forests protect land and water resources, filter water, regulate water regimes and help prevent land erosion and desertification. Fourth, forests provide economic, socio-cultural, aesthetic and recreational services, etc. In this paper we concentrate mainly on the role of forests as carbon sinks, even if the framework used here could be extended to include the other aspects.

We view forests as providers of competing economic and environmental goods. While forest logging brings economic revenues from both timber and agriculture on deforested land in the short run (FAO,

\footnotetext{
it The second author's research is partially supported by MEC under projects, ECO201124352, and ECO2014-52343-P, co-financed by FEDER funds, and COST Action IS1104. The third author's research is supported by NSERC, Canada.

* Corresponding author at: HEC Montréal, Canada.

E-mail addresses: pablo.andres.domenech@gmail.com (P. Andrés-Domenech), guiomar@eco.uva.es (G. Martín-Herrán), georges.zaccour@gerad.ca (G. Zaccour).
}

2006), excessive logging can exacerbate the problem of greenhouse gases (GHGs) accumulating in the long run. Reducing emissions from deforestation and degradation (REDD) has been put forward as a potentially cost-effective strategy to mitigate climate change. We build a model that accounts for GHG accumulation in the atmosphere in terms of anthropogenic emissions and carbon sequestration by the world's forests. The framework used allows one to: (i) evaluate the impact that forest depletion has on atmospheric GHG accumulation through the so-called reduced-carbon-sequestration effect, which states that a tree that is cut cannot grow and hence cannot sequester carbon; and (ii) compare the short-term rewards of high emissions and intensive deforestation policies with their long-term costs due to excessive GHG accumulation and forest depletion.

There is a significant dynamic-games literature dealing with the role of excessive GHG accumulation (see, e.g., the early papers of van der Ploeg and de Zeeuw (1992), Long (1992), Dockner and Long (1993) and the literature review by Jørgensen et al. (2010)). In this literature, emissions are a control variable and the issue is to determine the optimal emissions rate so as to reduce the environmental damage coming from the excessive accumulation of GHGs. Typically, these models concentrate on the difficulty of coordinating optimal emission levels and treat carbon sequestration as exogenously given. That is, as a constant fraction of the total stock of greenhouse gases. In this paper we model total carbon sequestration by forests explicitly and endogenously. If forests worldwide become rarer as a consequence of agents' decisions then 
the ability of worldwide forests to sequester carbon is reduced. A first contribution of this paper to this literature is in explicitly accounting for the role of forests as a carbon sink instead of just using an exogenous component that will yield the same amount of tons of carbon sequestered regardless of the state of the forests worldwide.

There is also a literature that deals with forest depletion using a dynamic-game approach (e.g., van Soest and Lensink (2000), Fredj et al. (2004, 2006), Martín-Herrán and Tidball (2005) and Martín-Herrán et al. (2006)). In these articles the players are forest owners, who exploit their asset to obtain economic revenues; and a donor community, or an environmentally aware player, who is willing to compensate forest owners who engage in preservation efforts of the resource.

We develop in this paper a model that merges these two strands of the literature. On the one hand, forest owners exploit (and eventually deplete) the forest. Their actions have an environmental impact on the atmospheric accumulation of GHGs. On the other hand, the nonforest-owner group derives utility from production (i.e., emissions) and disutility from the accumulation of GHGs in the atmosphere. In this setting, it is this disutility they experience that may eventually turn them into donors to preserve the forest as a carbon sink. This modeling framework allows us to capture both the high opportunity cost of reducing deforestation and the negative economic externality that forest owners inflict on non-owners as a consequence of their deforestation policy. Unlike the other aforementioned papers, we do not focus solely on forest conservation but also on its impact on GHG accumulation. In this sense, non-forest owners are also to decide on the best way to adjust their emissions.

The parameters of the model are empirically estimated, a rarity in the literature applying game theory to environmental problems. We believe that this constitutes a valuable contribution to the literature and an interesting demonstration case for policy and decision makers on how strategic interactions affect the evolution of both GHG accumulation and forest depletion. We determine the jointly optimal outcomes and compare them to the non-cooperative or business-as-usual counterparts. When the planning horizon is sufficiently long, then the cooperative solution is overall welfare improving. Cooperation partly reduces the negative externality and we analyze when it is profitable to abate emissions, when it is profitable to reduce net deforestation, and when it is optimal to do both (abate and reduce net deforestation). The results obtained show that it is preferable (cheaper) to invest in deforestation reduction rather than in emissions abatement when the perceived damages are low. However, as the environmental damages increase, it becomes optimal to combine emissions abatement with deforestation reduction.

Another aspect of key importance within the REDD literature is determining how these measures (i.e., measures involving emissions reductions) are to be implemented. In this paper we focus on the technical aspects related to the financial implementation of such policies within a dynamic setting: The cooperative solution brings economic gains, however these are asymmetric: the non-owner group gains while forest owners lose. Thus any environmental agreement attempting to implement the cooperative solution will require monetary compensation from the agents who win (non-owners) to the agents who lose (forest owners). Necessarily, forest owners have to be compensated with an amount at least equal to the difference between the sum of their intertemporal cooperative and non-cooperative payoffs. This compensation can be viewed as a payment for environmental services (PES) provided by forest owners. However, this requirement is not enough: When designing an intertemporal compensation mechanism (i.e., PES scheme), it is of key importance to allocate the transfers in such a way that no player has an economic incentive to deviate from the cooperative agreement at any instant of time, i.e., that the agreement be time consistent. We show that a division of joint payoffs using a dynamic Nash-Bargaining Scheme yields time-consistent outcomes.

The remainder of the paper is organized as follows: the model used for the two types of agents is presented in Section 2. In Section 3, the non-cooperative optimal policies for each player are obtained. Then, in Section 4, we compute the optimal cooperative policies and compare them to their non-cooperative counterparts. Section 5 is devoted to analyzing the feasibility and dynamic stability of the cooperative solution. Finally, all the results obtained are summarized in Section 6. The proofs are collected in the Appendices (Supplementary Material).

\section{The Model}

We consider two types of agents: forest owners and non-owners. Forest owners are modeled here as environmentally unconcerned agents who only care about revenues from deforestation, that is, they do not consider the consequences of their deforestation policy on GHG accumulation. Conversely, non-owners get revenues from the production of economic goods. ${ }^{1}$ Their productive activity generates emissions and this non-forest-owner group does take into account the negative effects of their current emissions policies on GHG accumulation in the atmosphere. This way of modeling allows us to capture the negative externality that forest owners create on the non-forest-owner group through the so-called reduced-carbon-sequestration effect.

We wish to state from the outset that our assumption that forest owners are environmentally unconcerned agents is undoubtedly a simplifying one. Still, it can be motivated on at least two relevant grounds, namely, methodology and realism. First, this assumption allows us to compute the upper-bound monetary compensation that is needed to induce forestry countries to save their forests. Accounting for any additional benefits of forests to their owners would decrease this value accordingly. Second, following many others, it has been argued by Masoudi and Zaccour (2013) that "compared to other pressing economic issues, such as eradicating extreme poverty, offering essential services to their citizens (education, health care, etc.) and building infrastructure, the environment is seen as a luxury service that developing countries cannot really afford in the short term." Considering that deforestation is mainly occurring in developing forestry countries, then our assumption reflects, albeit in a drastic way, this state of affairs.

\subsection{The Forest Owners' Problem}

Forest owners maximize their discounted stream of net revenues, which depend on their afforestation and deforestation rates, $A(t)$ and $D(t)$ respectively, as well as on the existing forest surface area $F(t)$ measured in hectares. Net revenues are discounted at rate $r$ throughout a finite-time horizon, denoted $T$. In the next section, we let the planning horizon vary and show how the optimization results depend on the value of $T$.

Net revenues include gross revenues $R(t)$, afforestation costs $\kappa_{1} A(t)$ and deforestation costs $\kappa_{2} D(t)$, where $\kappa_{1}$ and $\kappa_{2}$ are respectively the per-hectare afforestation and deforestation costs. The forest owners' objective is the following:

$\max _{A(t), D(t)} \int_{0}^{T} e^{-r t}\left[R(t)-\kappa_{1} A(t)-\kappa_{2} D(t)\right] d t$,

where $A(t) \in\left[0, A_{\max }\right]$ and $D(t) \in\left[0, D_{\max }\right]$. The upper bounds for afforestation $\left(A_{\max }\right)$ and deforestation $\left(D_{\max }\right)$ reflect the idea that there is a physical limit, in the short term, to afforestation and that deforestation is subject to a regulation that allows for it within certain limits. The value of $D_{\max }$ is set to fit the observed world deforestation figures

\footnotetext{
${ }^{1}$ Although it may be interesting in some cases to understand these two players as blocks of countries (i.e. developing and developed), we prefer to use the current terminology (owners and non-owners) for it does not preclude the case where a non-owner is the citizen of, say, São Paulo and a forest-owner another Brazilian citizen in, say, the Amazonia. Likewise we are not very fond of the dichotomy owners-consumers because it somehow presupposes that owners do not consume and that consumers only care about consuming. Admittedly, the terminology that we use is not the simplest, but we thought that it was the richest in interpretations and wanted to leave this matter open.
} 
provided by the FAO (2006). The definitions of all parameters, their values and their sources are provided in Appendix A.

We assume that the evolution over time of the forest area can be well approximated by the following linear differential equation:

$\dot{F}(t)=A(t)+\eta F(t)-D(t), \quad \bar{F} \geq F \geq 0, \quad F(0)=F_{0}$,

where $\eta$ is a positive parameter, $\bar{F}$ is the maximum surface suitable to forest colonization and $F_{0}$ is the initial forest world's surface area in 2005 (FAO, 2006), i.e., nearly 4 billion ha. Eq. (2) is an extension of van Soest and Lensink (2000) and Fredj et al. (2006), where $A=\eta=$ 0 in the first and $A=0$ in the second. Note that the linear specification in Eq. (2) approximates reasonably well forest expansion within a large interval around the current world forest area $F(0)$.

Forest owners obtain revenues from selling timber and agricultural products. Denote by $q(t)$ the quantity of timber put on the market at time $t$, and let the price $p(t)$ be given by the following inverse demand function:

$p(t)=\bar{p}-\theta q(t)$

where $\bar{p}$ is the choke price that sets demand equal to zero, and $\theta$ its slope. The values of parameters $\bar{p}$ and $\theta$ have been calibrated using data given by FAO on timber prices and quantities.

The quantity $q(t)$ comes from two different sources, namely, clearfelling and selective logging, and is given by

$q(t)=y D(t)+y \gamma \delta F(t)$,

where $y D(t)$ is the amount of wood retrieved from clear-felling an area $D(t)$ and the product $y \gamma \delta F(t)$ stands for the total selective-logging yield, which is lower (in per-hectare terms) than the yield obtained through clear-felling. Parameter $y$ denotes the per-hectare timber yield and is typically measured in stems per hectare or cubic meters of timber per hectare. FAO (2006) provides an estimate for this parameter. Clearfelling an area $D(t)$ reduces the total forest size by the same amount. However, unlike deforestation, selective logging is assumed to have no impact on total forest land. According to FAO, "[selective logging]...is not necessarily destructive and can be done with low impact on the remaining forests, if the proper techniques are applied". ${ }^{2}$ Clearly, for selective logging to have a negligible environmental impact, its per-hectare yield per unit of area must be much lower than the clear-felling one. This lower yield is accounted for by parameter $\gamma(\gamma<<1)$. Finally, according to FAO (2006), roughly one-third of the world's forests are used primarily for the production of wood and non-wood forest products. Parameter $\delta$ takes into account the fact that only a fraction of the world's forests are actually being exploited. ${ }^{3}$

Agriculture revenues are equal to the prices times the yields of the different crops grown. For simplicity, we suppose that forest owners grow a single agricultural good, which we model as a composite good made of four representative crops that are commonly related to deforestation processes. This good is sold in international markets at a given price $p_{A}{ }^{4}$ The total yield at time $t$ depends on the size of the available (previously deforested) land, given by $\bar{F}-F(t)$, where $\bar{F}$ stands for the maximum size or carrying capacity of the forest, and on the soil productivity $x(t)$.

Putting together the revenues from timber sales and agricultural products, we get the following expression for gross revenue:

$R(t)=p(t) q(t)+p_{A} x(t)(\bar{F}-F(t))$.

\footnotetext{
2 Source: http://www.fao.org/forestry/news/48681/en/.

3 In van Soest and Lensink (2000), the parameters $\gamma$ and $\delta$ are set equal to one. Here we follow the more general specification used by Andrés-Domenech et al. (2011).

${ }^{4}$ The price $p_{A}$ is constant, unlike $p(t)$, due to the fact that agricultural production on deforested land represents only a fraction of the world's total agricultural land.
}

As in Andrés-Domenech et al. (2011)-see also van Soest and Lensink (2000) for a simpler version-we model $x(t)$ as follows:

$x(t)=\bar{x}+\alpha(t) D(t)-\beta \frac{\bar{F}-F(t)}{\bar{F}}$.

The above expression of the total productivity of land $x(t)$ is the sum of three terms. The first is a constant productivity term $\bar{x}$ that measures the average yield in tons of crop per hectare of land for a representative agricultural good. The second term, $\alpha(t) D(t)$, captures the idea that newly deforested land $D(t)$ is more productive. Variable $\alpha(t)$ measures the increase in the total average per-hectare production resulting from deforesting an area $D(t)$. The third term, $-\beta \frac{\bar{F}-F(t)}{\bar{F}}$, accounts for the positive externality that forests generate on nearby agricultural land. Forests are seen as a source of rain and a protective element for agricultural land. Parameter $\beta$ measures the decrease (increase) in soil quality and, therefore, in agricultural productivity caused by forest depletion (expansion). The productivity increase of newly deforested land is given by

$\alpha(t)=\frac{\psi \bar{x}}{\bar{F}-F(t)}$

Newly deforested land is more productive and parameter $\psi$ measures the factor by which productivity is increased. However, this extra productivity needs to be normalized for all agricultural land. We divide the extra yield, $\psi \bar{x}$, by the total agricultural surface area, $\bar{F}-F(t)$, otherwise the term $\alpha(t) D(t)$ in Eq. (6) would overestimate the real impact that deforesting an area $D(t)$ has. $^{5}$

To recapitulate, forest owners maximize their net discounted economic revenues Eq. (1) with respect to their deforestation and afforestation efforts, $D(t)$ and $A(t)$, respectively, and subject to the forest dynamics in Eq. (2).

\subsection{The Non-owners' Problem}

Non-owners optimize a two-part objective function. The first part consists of a short-run gain derived from producing and consuming economic goods. The production of these goods generates pollution as a byproduct and this pollution affects their utility. For simplicity, we suppose that the carbon intensity of the economy is constant. Hence, ceteris paribus, producing more goods is equivalent to emitting more. ${ }^{6}$ The second term in the objective of the non-forest-owner group represents an economic loss or damage related to the accumulation of emissions in the atmosphere. We will specify the functional forms of these two terms after describing the dynamics of the GHGs emissions and the stock of pollution.

Denote by $E(t)$ the GHGs emissions by the non-forest-owner group. Emissions, in our model, are assumed to be exclusively anthropogenic and are given entirely by the emissions of the non-forest-owner group. By this we do not mean that forest owners do not emit but rather that their contribution to global emissions is negligible. ${ }^{7}$ The dynamics of the emissions rate $E(t)$ is then given by

$$
\dot{E}(t)=V(t) E(t), \quad E(t) \geq 0, \quad E(0)=E_{0}
$$

\footnotetext{
${ }^{5}$ Agricultural revenues are obtained by multiplying the productivity (6) by total agricultural land. Hence Eq. (6) has to account for average per-hectare productivity measured in tons of crop per hectare. For this reason, the term $\alpha(t) D(t)$ cannot be understood as the extra productivity of newly deforested land, but rather as the normalized productivity increase that newly deforested land has on total agricultural land.

${ }^{6}$ One could think of a more refined formulation, where the economy's carbon intensity can adjust, and where production increases can be compatible with constant emissions levels or even decreases.

${ }^{7}$ If forest owners' emissions were to be included explicitly in expression (8) the model would still be solved in the same way.
} 
The dynamics of emissions in Eq. (8) can also be written in a more familiar way, that is,

$$
\frac{\dot{E}(t)}{E(t)}=V(t)
$$

where $V(t)$ denotes the instantaneous speed of emissions variation, which is the non-owners control variable. Non-owners maximize their payoffs by adjusting their emissions, and their decision has an impact on the state of the system. For the sake of realism $V(t)$ has been modeled as a bounded control variable (i.e., $V_{\min } \leq V(t) \leq V_{\max }$ ), with $V_{\min }<0$ and $V_{\max }>0$. In the literature, it is more common to see emissions as a flow variable. Our modeling of emissions allows us to better account for the inertia of the productive and economic systems. Indeed, emissions take time to adjust and the upper and lower bounds on $V(t)$ simply reflect this idea that emissions cannot be increased or decreased at whatever rate. One can think of these bounds as being given by the existence of technical, economic and/or political constraints.

The evolution of the stock of greenhouse gases in the atmosphere depends on emissions and on carbon sequestration by the world's forests and oceans. Forests worldwide sequester carbon as they grow, and according to the IPCC (2000) and FAO (2006), approximately half of the dry weight of forest biomass is carbon. To model carbon sequestration by forests, one could measure the variation in the total forest biomass; however, this would present two main difficulties. First, the variation in total carbon biomass is difficult to measure. And second, measuring carbon sequestration through the variation in forest biomass underestimates the total carbon sequestration since timber captures are neglected. To overcome this problem, we make the simplifying assumption that forest owners manage a representative forest whose trees grow-volume wise-at an average and constant rate. Having a representative forest whose growth rate is constant allows us to express carbon sequestration as a linear function of forest area alone (i.e., carbon sequestered per hectare of forest land and per unit of time). The advantage of having carbon sequestration in terms of forest area rather than in terms of biomass variation is that one can easily consider timber captures, while gaining a tractable and understandable way to measure carbon sequestration.

Further, note that by measuring carbon sequestration as a function of the forests' surface area, one can account for the so called reducedcarbon sequestration effect, which is based on the simple principle that a tree that is cut cannot grow (i.e., cannot sequester carbon). Thus, it is straightforward to see that deforestation has a negative impact on carbon sequestration due to the reduction in forest area that it induces. Expression (9) below captures the dynamics of the atmospheric concentration of carbon in terms of the forest stock, where parameter $\phi$ reflects the amount of carbon sequestered per hectare of forest and per unit of time.

We also consider the oceans as a second type of carbon sink. Denoted by $S(t)$ the stock of GHGs (e.g., stock of $\mathrm{CO}_{2}$ ) in the atmosphere and by $W(t)$ the amount of carbon that the oceans sequester at a given time $t$. The carbon uptake by the oceans has remained relatively stable during the last few years, and for this reason, $W$ has been assumed constant for simplicity's sake even if there exist small year-to-year variations due to El Niño effects (Le Quéré et al. (2009)). The evolution of the stock of pollution is then given by the following differential equation:

$\dot{S}(t)=E(t)-\phi F(t)-W, \quad S(t) \geq 0, \quad S(0)=S_{0}$.

Once the time evolution of the emissions rate and the stock of pollution have been described, we come back to the description of the non-owners' two-part objective function. The first part concerns the payoff generated in terms of goods production and is described by the concave increasing function $G(E)$. We adopt the following functional form:

$G(E(t))=a E(t)-\frac{1}{2} b E^{2}(t)$

where parameters $a$ and $b$ are positive and have been fixed in order to ensure that $G^{\prime}(E)>0$ for the relevant range of emissions. This specification is similar to the one proposed in, e.g., Dockner and Long (1993) and Breton et al. (2005), with the only difference being that we have included parameter $b$ to calibrate $G(E(t))$ at the current world-level GDP.

The second term in the objective of the non-forest-owner group represents an economic loss or damage related to the accumulation of emissions in the atmosphere. According to the IPCC (2007), increases in the atmospheric concentration of GHGs result in seawater levels rising, temperatures increasing and seawater acidification. These processes are all related to economic and environmental damage. We assume that the damage cost is given by a strictly convex and increasing function $L(S)$, with $S$ the stock of GHGs (e.g., stock of $\mathrm{CO}_{2}$ ) in the atmosphere. Although we acknowledge the existence of thresholds, extreme events and jumps in the damage, ${ }^{8}$ our formulation, which is very common in the literature (see, e.g., Benchekroun and Long (2002), Dockner and Long (1993), van der Ploeg and De Zeeuw (1992), Breton et al. (2006)), smooths the impacts of such phenomena rather than dealing with them explicitly. Needless to say, accounting properly for non linearities and threshold effects in the damage cost would lead to a model of much greater complexity.

That being said, for a specific function to qualify as a good candidate to model such damages we can think of yet another necessary requirement: greenhouse gases, and most particularly $\mathrm{CO}_{2}$, have always been present in the atmosphere and represent a basic element for the existence and development of life (e.g., plants). It is clear that it is not the presence, but the excessive accumulation of atmospheric GHGs that poses the problem. We adopt the following specification of $L(S)$ to capture all these elements in a simple way:

$L(S(t))=c(S(t)-\underline{S})^{2}$,

where $\underline{S}$ is a natural threshold, beyond which the GHG concentration is considered excessive and economic and environmental damages are perceived. In practical terms, choosing a reasonable value for $\underline{S}$-given the above specification-amounts to choosing a level of atmospheric GHGs for which there is no perceived damage. We identify $\underline{S}$ with the pre-industrial level of GHGs (see, e.g., Bahn et al. (2008)).

Taking into account the gain function $G(E)$ and the damage function $L(S)$, we obtain the following objective functional, which is maximized by the non-forest-owner group:

$\int_{0}^{T} e^{-r t}[G(E(t))-L(S(t))] d t-\phi(S(T)) e^{-r T}$,

where $r$ is the market discount rate (the same as for forest owners), and $\phi(S(T))$ is a salvage value.

Non-owners are modeled as forward-looking agents who consider the long-term impact of their decisions. The stock of emissions accumulates slowly and then has a long-term impact on non-owners' payoffs. Therefore, it is sensible to have a scrap-value function somehow related to the stock of emissions at the terminal date of the planning horizon. Such a salvage-value function can be generically written as $\phi(S(T))$. It is reasonable to think that, whatever the GHG stock at the terminal date, it will strongly impact future payoffs due to the long-term

\footnotetext{
8 For instance, a small increase in the atmospheric concentration of GHGs can bring a quantitatively different damage but may also trigger qualitatively different damages (e.g., massive ice-cap melting, dissolution of coral reefs as a result of extreme oceanic acidification)
} 
persistence of greenhouse gases in the atmosphere. One could think of a more sophisticated scrap-value function that also depends on the final forest stock or on the emissions policy followed after the terminal date, or even define the scrap-value function as an identical problem to the one presented above in Eq. (12). Because we want to keep the model parsimonious, and because we want to be able to say something that is irrespective of what policies are chosen after the terminal date, we have chosen the following formulation for $\varphi(S(T))$, which depends on the terminal stock of greenhouse gases alone:

$\phi(S(T))=\int_{T}^{2 T} e^{-r(s-T)} L(S(T)) d s$.

Although the salvage function in Eq. (13) is simple, it satisfies the following desirable requirements: (i) it reflects the idea that the terminal stock of GHGs matters and has an impact on future payoffs; (ii) it is easy to compute and does not depend on (potentially) unknown future policies; (iii) it keeps discounting in a natural way the cost of future environmental damages; and (iv) the time span considered for the scrap value function is related to the planning horizon. In fact, if the planning horizon chosen is short, then the weight given for future environmental damages will likely be small as well and vice versa.

To wrap up, non-owners maximize their payoff in Eq. (12) adjusting the instantaneous variation of emissions $V(t)$, subject to Eqs. (8) and (9) and given the fact that the solution to Eq. (2) is inherited from the forest owners' problem.

\section{Individual Optimization}

In this section we characterize the optimal strategies of the two players when they act independently. As the forest owners' payoffs do not depend on emissions or on GHG accumulation, their payoffs are independent of the action of non-owners. On the other hand, non-owners' payoffs are affected by the forest owners' decisions through the evolution of the forest stock. In this setting, where there is a one-way interaction, Nash and Stackelberg equilibria coincide. Further, open-loop and feedback-information structures yield the same result. Given this, we can first solve the economic problem of the forest owners, and next, optimize for the non-forest-owner group, taking the evolution in the forest stock as given.

In the rest of the paper we use $\mathcal{O}$ to denote the forest owners and $\ominus$ to refer to the non-owners.

\subsection{Forest Owners}

Forest owners maximize their revenues in Eq. (1) subject to Eqs. (2)-(5). The following proposition provides the optimal solution to their control problem (the superscript $n c$ stands for noncooperation).

Proposition 1. For the parameter domain defined in Appendix A, the optimal control, state and co-state variables are given by ${ }^{9}$

$$
\begin{aligned}
A^{n c}(t) & =0, \quad D^{n c}(t)=D_{\max } \text { for all } t \in[0, T] \\
F(t)= & \left(F_{0}-\frac{D_{\max }}{\eta}\right) e^{\eta t}+\frac{D_{\max }}{\eta}, \\
\lambda(t)= & \frac{1}{\eta-r}\left[1-e^{(\eta-r)(T-t)}\right] \\
& \times\left\{\left(2 \theta y D_{\max }-\bar{p}\right) y \gamma \delta+p_{A}(\bar{x}-2 \beta)+2 F(t)\left[\theta y^{2} \gamma^{2} \delta^{2}+p_{A} \beta \frac{1}{\bar{F}}\right]\right\} .
\end{aligned}
$$

\footnotetext{
9 The second-order sufficient optimality conditions are satisfied for this and all the problems studied in this paper.
}

\section{Proof. See Appendix B.}

The results show that the forest owners' optimal strategy consists in deforesting at maximum admissible level and not afforesting at all. As the problem is linear in the afforestation effort, and afforestation is a pure cost in our setting, then the optimal strategy is obviously to set $A(t)$ at its lowest admissible value, i.e., $A(t)=0$. Further, the marginal revenue from agricultural activity is positive for all admissible values of $D(t)$, including $D_{\max }$. Therefore, there is an incentive to deforest at the maximum level. ${ }^{10}$ These results follow from the fact that, for our parameter domain, we have $\lambda(t) \leq 0$, for all $t$. Indeed: (i) the term $\frac{1}{\eta-r}\left[1-e^{(\eta-r)(T-t)}\right]$ is always negative since $\frac{1}{\eta-r}$ and $\left[1-e^{(\eta-r)(T-t)}\right]$ are of opposite sign, regardless of the values of $\eta$ and $r$; and (ii) $(2 \theta y D(t)-\bar{p}) y \gamma \delta+p_{A}(\bar{x}-2 \beta)>0$, for all admissible values of $D(t)$, including $D_{\text {max }}$. Deforestation is mainly driven by the revenues obtained from growing agricultural products on deforested land, rather than by the timber revenues that arise from deforestation itself. This is in line with other studies, e.g., Barbier and Rauscher (1994), Barbier and Burgess (2001) and FAO (2006), which suggested that deforestation for agricultural purposes is the main explanatory factor for forest depletion worldwide.

\subsection{Non-owners}

The non-owners maximize their payoff given by Eq. (12) and take into account the values of the three state variables, namely, forest area, $F$, emissions, $E$, and the stock of accumulated emissions in the atmosphere, $S$. The optimal solution depends on the length of the planning horizon and on the intertemporal discount rate. For the values of our parameters, the solution is constant $\left(V^{n c}=V_{\max }\right)$ as long as the planning horizon $(T)$ is less than approximately forty years, (i.e., $T \lesssim 40) .{ }^{11}$ The following proposition provides the optimal solution to the problem of non-forest owners and the optimal time paths for control and state variables in such a case.

Proposition 2. For the parameter domain defined in Appendix A and $T \lesssim 40$, the optimal control and state variables are given by

$$
\begin{aligned}
V(t) & =V^{n c}=V_{\max } \quad \text { for all } \quad t \in[0, T] \\
E(t) & =E_{0} e^{V^{n c} t} \\
S(t) & =S_{0}-\frac{\varphi}{\eta} t D_{\max }-\frac{E_{0}}{V^{n c}}\left(1-e^{V^{n c} t}\right)+\frac{\varphi}{\eta}\left(F_{0}-\frac{D_{\max }}{\eta}\right)\left(1-e^{\eta t}\right) .
\end{aligned}
$$

\section{Proof. See Appendix C.}

As pointed out above, the optimal control $V^{n c}$ depends on the planning horizon being considered. For a relatively short horizon, i.e., $T \lesssim 40$, the optimal solution is constant of the type $V^{n c}=V_{\max }$ all along. The solutions shown in Proposition 2 hold for as long as there is no switching time. For longer horizons, i.e., $T \gtrsim 40$, the optimal solution is to apply the control $V=V_{\max }$ for some time and then switch to a cleaner regime. ${ }^{12}$ For much longer horizons (i.e., $T>100$ ), it is possible that the optimal solution consists of switching not once but several times. In all cases, the different switching times and the number of

\footnotetext{
${ }^{10}$ Note that these results are mainly driven by the fact that forest owners-as they are modeled here-do not internalize the environmental costs of their actions. If they did take into account the negative impact of deforestation on the atmospheric accumulation of GHGs the optimal afforestation and deforestation rates would be lower.

11 The determination of the exact planning horizon beyond which Proposition 2 does not hold depends on the intertemporal discount rate used. As we will see, for every value of the discount rate, we can obtain the maximum value of $T$ for which Proposition 2 holds.

12 It can be shown that singular arcs are not possible.
} 
Table 1

Sketch of algorithm used to compute the optimal switching time $\tilde{t}_{V}$.

Fix the length of the planning horizon $(T)$ and the discount rate (r) for all possible integer switching times $\left(t_{V}\right)$ do

$$
\begin{aligned}
\operatorname{Payoff}\left(t_{V}\right) & =\text { Discounted sum of payoffs before switch } t_{V} \\
& + \text { Discounted sum of payoffs after switch } t_{V} \\
& + \text { Scrap-value function }
\end{aligned}
$$

end

Select the $t_{V}$ whose Payoff is greatest

switches depend on the value adopted for $T$. Denote by $\tilde{t}_{V}$ the optimal switching time. Then, the optimal solution for $40 \lesssim T \leq 100$ can be summarized as follows:

$V(t)=\left\{\begin{array}{ll}V_{\max }, & \text { for } \leq \tilde{t}_{V} \\ V_{\min }, & \text { fort }>\tilde{t}_{V}\end{array}\right.$.

In Appendix D we have solved the problem for the case where there is only one switching time, and characterized the first-order conditions that apply in that case. Retrieving the actual switching time, however, represents a challenge. This is mainly due to the change in the evolution of the state and co-state variables as a consequence of changes in the switching time itself. The first-order conditions before and after the switch will only be satisfied if the exact switching time is chosen. This poses a problem in determining the actual switching time since one has to try an infinite number of possibilities, and the first-order conditions will only be satisfied if the exact one is chosen.

To overcome this problem we have developed an algorithm to obtain the optimal switching time, approximated to the integer value (time) at which it is best to switch. The proposed algorithm consists of evaluating the sum of the payoffs for all possible scenarios (i.e., all possible switching times). From them, we then select the integer time for which shifting regime (from $V_{\max }$ to $V_{\min }$ ) yields the greatest payoffs. A sketch of the algorithm can be found in Table 1.

Suppose for instance that our planning horizon and discount rate were fixed at, e.g., $T=50, r=0.02$. Fig. 1 gives the payoffs of the non-forest-owner group in the $y$-axis for each possible switching time $\left(x\right.$-axis). We observe, for this particular case, that switching from $V_{\max }$ to $V_{\text {min }}$ after $\tilde{t}_{V}$ (where $\tilde{t}_{V}=17$ years) is the best course of action.

We can generalize the algorithm presented in Table 1 and let the planning horizon $T$ vary while keeping the discount rate $r$ constant. In so doing we obtain the best switching time for each different planning horizon.

Fig. 2 gives the optimal switching time for each possible planning horizon $T$. To better understand this figure, it is important to distinguish between three elements, namely,

$T$ : the planning horizon;

$T^{s}$ : the minimum planning horizon for a switch to take place; $\tilde{t}_{V}$ : the actual switching time.

The diagonal line indicates that no switch is applicable. The shortest planning horizon for which there is a switch, $T^{s}$, is the first element of the curve off the diagonal. Fig. 2 illustrates the fact that it pays to emit more in the short run (i.e., it is optimal to increase emissions first to reduce them later and not the opposite). It also shows that for longer planning horizons it is comparatively less attractive to apply $V_{\max }$. This result is related to the non-linear damage function $L(S)$, by which the environmental damage increases when GHGs accumulate due to excessive emissions, on the one hand; and to the effect of increasing the damages accounted for by increasing the planning horizon, on the other.

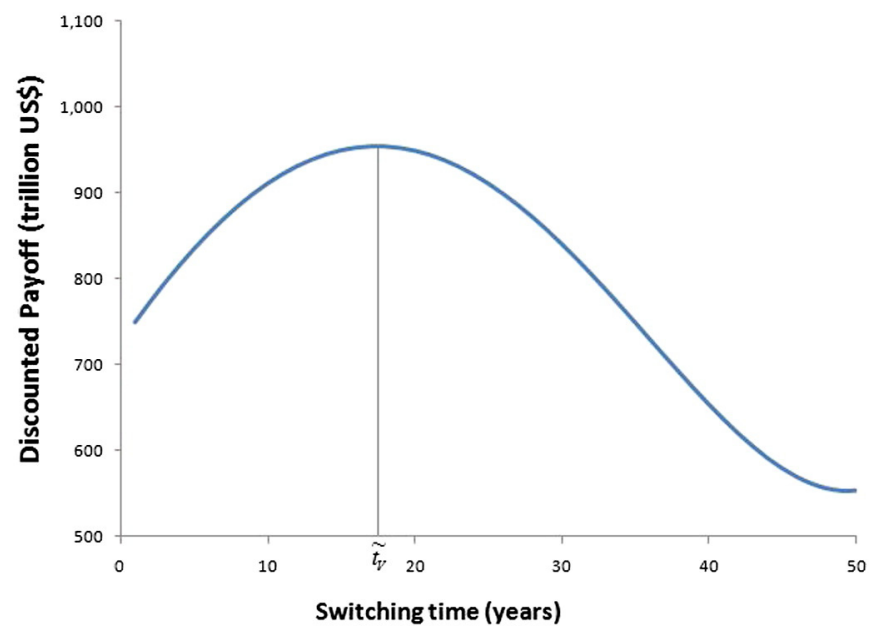

Fig. 1. Payoffs as a function of the switching time.

In Fig. $2, T^{s}=38$. This means that there is no switch if $T<38$, and that there will be one if $T \geq T^{s}$. As mentioned before, $T^{s}$ and $\tilde{t}_{V}$ do not coincide, even when $T=T^{s}$. Put differently, if the planning horizon is long enough the non-forest-owner group recognizes the need to switch to a cleaner regime, but the switch will take place some time before the terminal date. Note that the pair $\left(T=50, \tilde{t}_{V}=17\right)$, which we obtained in Fig. 1, is now just one point of the curve displayed in Fig. 2.

We can further generalize our algorithm for any value of $r$. In the previous two figures, $r$ was set equal to 0.02 (2\%). The previous results are compared with two other alternative scenarios, $r=1 \%$ and $r=$ 3\%, in Fig. 3.

Fig. 3 conveys a dual message: First, when the discount rate is lower, the non-owners internalize the negative externality earlier, due to the accumulation of GHGs in the atmosphere. This can be inferred from the fact that $T^{s}$ is lower for lower discount rates. In particular, we have that $T^{s}=35$ if $r=1 \%$; $T^{s}=38$ if $r=2 \%$; and $T^{s}=41$ if $r=3 \%$. Second, regardless of the discount rate, the longer the time horizon used, the earlier the switch, i.e., the three curves are downward sloped.

To summarize, it is optimal for the non-owners to increase emissions if $T \lesssim 40$. If $T \gtrsim 40$, it is better to switch to a cleaner regime $\left(V=V_{\min }\right)$ at some time $\tilde{t}_{V}$. The optimal time for the switch directly depends on the planning horizon and the discount rate used. A simple folk conjecture says that the longer the planning horizon and/or the smaller the intertemporal discount rate, the sooner this switch will arrive. This is related to the damage function $L(S)$, which yields greater (cumulative) losses for lower discount rates and longer planning horizons.

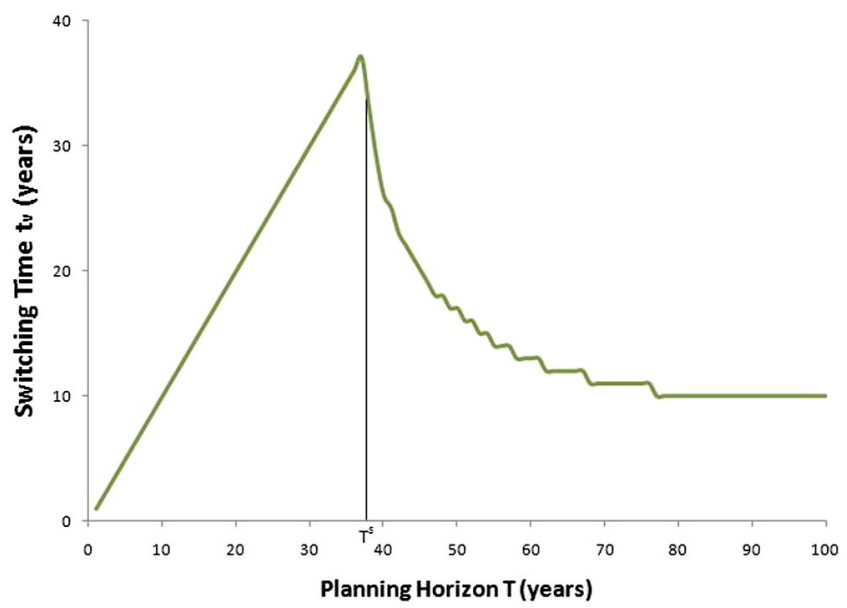

Fig. 2. Optimal switching time for every planning horizon. 


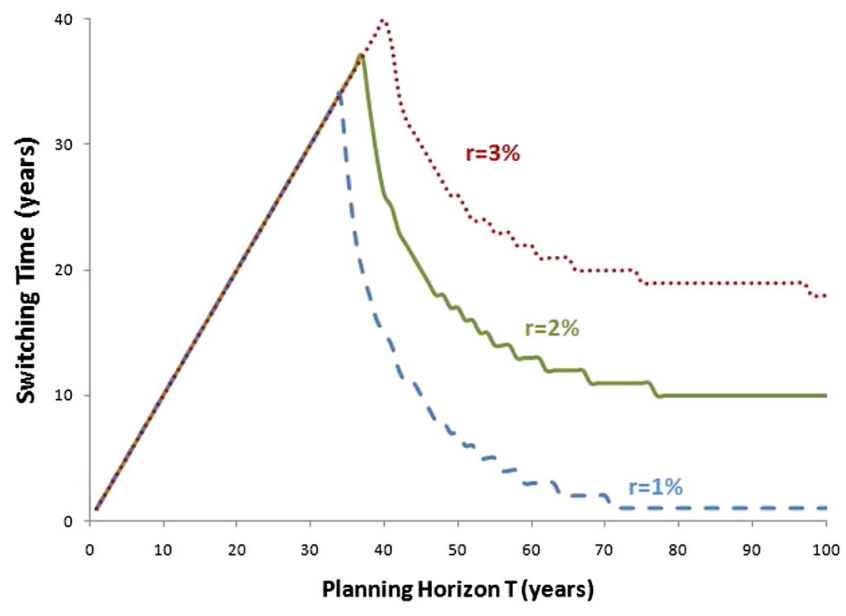

Fig. 3. Impact of the discount rate on the switching time.

It is worth noting at this point that we have no prescription to make in terms of what planning horizon should be used, we simply illustrate how the optimization results change as a function of the planning horizon. If anything, what our results suggest is that taking into account long-term damages may lead to sensibly different optimal solutions, in this sense it seems desirable to use a longer planning horizon.

It has been shown how to determine the switching time. To put things into perspective, denote by $\Pi$ the discounted sum of instantaneous payoffs over the planning horizon. The instantaneous payoff can be expressed in terms of the control $\pi(V)$ and so can $\Pi$ :

$\Pi(V(t))=\int_{0}^{T} e^{-r t} \pi(V(t)) d t-\phi(S(T)) e^{-r T}$.

One can compare the difference in the sum of discounted payoffs for non-owners when the optimal emissions trajectory (with switch), $\Pi$ $(\hat{V})$, is applied all along with the payoffs $\Pi\left(V_{\max }\right)$ and $\Pi\left(V_{\min }\right)$ that are obtained by applying the constant (and sub-optimal) solutions $V=V_{\max } \forall t \in[0, T]$ and $V=V_{\min } \forall t \in[0, T]$, respectively. The value of $\Pi(\hat{V})$ in Fig. 4 is obtained by computing expression (18) for $r=2 \%$ and for $T \in[0,100]$ along the optimal path for $E(t)$ and $S(t)$. Note that for $T<38 \Pi(\hat{V})$ and $\Pi\left(V_{\max }\right)$ coincide. For $T \geq 38$ there will be a switch and $\Pi(\hat{V})$ diverges from $\Pi\left(V_{\max }\right)$.

So far we have analyzed the optimal emissions policy. It is also important to analyze the sign of the shadow price of the forest stock, $\lambda_{F}$. This shadow price is positive regardless of the time horizon and

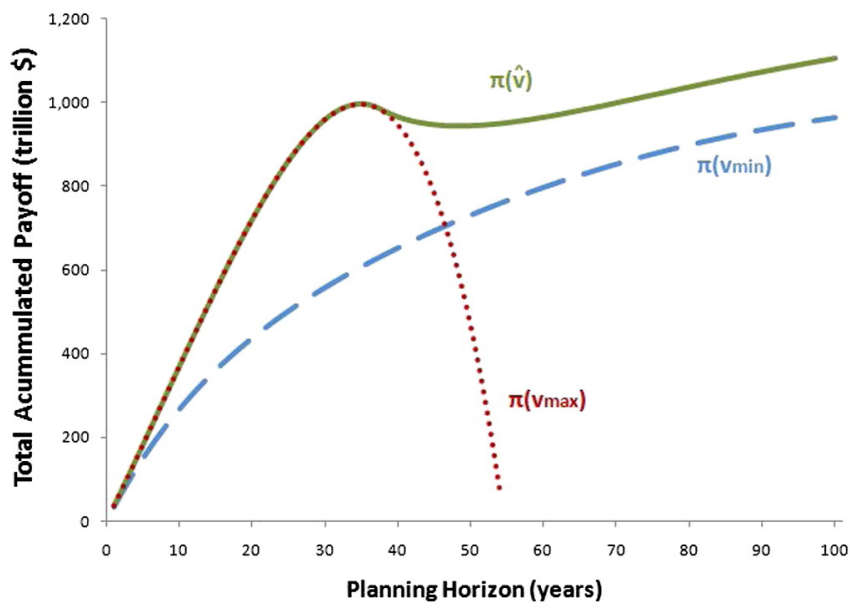

Fig. 4. Comparing $\Pi(\hat{V})$ with $\Pi\left(V_{\min }\right)$ and $\Pi\left(V_{\max }\right)$. discount rate considered. The positive sign of the co-state $\lambda_{F}$ is directly related to the ability that forests have to sequester carbon. Since the increase in forest area is directly related to the enhancement of carbon sequestration (see expression (9)); then, regardless of the value of $F$ and $S,{ }^{13}$ marginally increasing the forest area implies marginal reductions in $E(t)$, meaning smaller environmental losses (see expression (11)). This is a qualitative aspect.

At the same time, we have seen that the importance of reducing emissions is directly related to the length of the planning horizon and inversely related to the discount rate. Likewise, the marginal value that non-owners attach to an additional hectare of forest is greater when the planning horizon is longer and the discount rate is lower. This is a more quantitative aspect.

In short, unlike forest owners, the non-owners are interested in increasing the total forest area, and this is reflected by the sign of $\lambda_{F}$. If we compare the different ways in which forest owners and nonowners evaluate an additional hectare of forest, it is clear that there exists an environmental externality. Recall that forests in our model have at least two uses: (i) the provision of economic revenues; and (ii) carbon sequestration. These uses are competing and somewhat mutually excluding. Forest owners create a negative externality on non-owners with their net deforestation policy. Hence the question is: should this negative externality be corrected?

Given the existing property rights over the forest, and the fact that forest owners' payoffs are a decreasing function of the total forest area, reducing the net deforestation is harmful for forest owners. Therefore, the answer to this question depends on whether an additional unit of forest can generate an increase in the payoff of the non-owners, such that it more than compensates for the reduction in the forest owners' revenues when they apply a more environmentally friendly deforestation/afforestation policy. If that is the case, then it will be jointly optimal to correct the externality, or at least part of it. In the next section we compute joint payoffs to answer the question raised above. We also compare the cooperative scenario to the status-quo individual equilibrium results.

\section{Cooperative Solution}

In the previous section we determined the non-cooperative (statusquo) strategies for both forest owners and non-owners. We saw that forest owners find it optimal to deforest as much as possible and to not afforest. On the other hand, non-owners suffer a negative environmental externality coming from the depletion of the forest via the reduced-carbon-sequestration effect. A relevant question to address is whether cooperation can improve welfare. The collectively optimal solution can be obtained by jointly optimizing the payoff functionals of the two players, that is,

$$
\begin{array}{ll}
\max _{\substack{0 \leq A(t) \leq A_{\max } \\
0 \leq D(t) \leq D_{\max }, V_{\min } \leq V(t) \leq V_{\max }}} \int_{0}^{T} e^{-r t}[R(F(t), D(t))+G(E(t))-L(S(t))] d t-\varphi(S(T)) e^{-r T} . \\
\text { s.t.: } \quad \dot{F}(t)=A(t)+\eta F(t)-D(t), \quad \bar{F} \geq F(t) \geq 0, \quad F(0)=F_{0}, \\
& \dot{E}(t)=V(t) E(t), \quad E(t) \geq 0, \quad E(0)=E_{0}, \\
& \dot{S}(t)=E(t)-\phi F(t)-W, \quad S(t) \geq 0, \quad S(0)=S_{0},
\end{array}
$$

where $A, D$ and $V$ are the three control variables. The joint payoff is maximized subject to the dynamics of the forest area, emissions, and stock of greenhouse gases in the atmosphere.

Appendix E presents the optimality conditions for this cooperative problem.

As expected the optimal afforestation rate and speed of adjustment of emissions are bang-bang policies because the Lagrangian is linear in

\footnotetext{
${ }^{13}$ Clearly we are referring here to values of $S$ above $\underline{S}$.
} 
Table 2

Sketch of the algorithm used to obtain $\tilde{t}_{A}^{c}, \tilde{t}_{D}^{c}, \tilde{t}_{V}^{c}$.

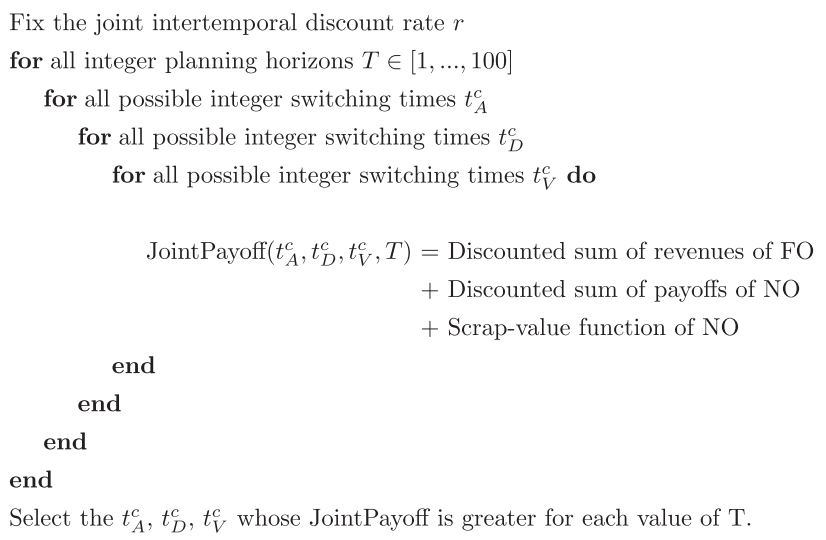

these variables. Further, although the co-state variable associated with the forest stock, $\lambda_{F}^{c}$, appears, as in the non-cooperative case, in the optimality conditions for $A$ and $D$, there is an important difference, that is, $\lambda_{F}^{c}$ now captures the negative valuation of an extra hectare of forest (forest owners) and the positive effect that increasing forest area has on carbon sequestration (non-owners). Therefore, $\lambda_{F}^{c}$ can take either positive or negative values depending on which effect dominates. Furthermore, unlike in the non-cooperative case where the sign of $\lambda_{F}$ was constant along the planning horizon for both players, now nothing prevents this sign from changing over time. Hence, it is possible that we have a switch in either the afforestation rate or the deforestation rate or in both throughout the planning horizon.

To solve for $\lambda_{F}^{c}$, we need the analytical expression for $F$, which depends on both $A$ and $D$ (see (E.1)). In the non-cooperative scenario it was possible to analytically characterize the solution to the forest owners' problem by supposing ex-ante that we were in the right case of figure, and then verifying, ex-post, that our first-order conditions were indeed satisfied (see Appendix B for more details). This reasoning was possible because the optimal afforestation and deforestation rates were constant. In the present case however, we can have a policy switch on $A$ and/or $D$ at any time. Therefore, the value of $\lambda_{F}^{c}$ depends on the switching time on $A$ and $D$. The implication is that the first-order conditions will be satisfied for all $t \in[0, T]$ only if the exact switching time for both variables is chosen.

From (E.3) we see that $\lambda_{E}^{c}$ depends on $\lambda_{S}^{c}$, and from (E.2) we have that $S$ is a function of $F$. Therefore, to obtain $\lambda_{E}^{c}$ we need to know the evolution of the forest stock, which depends on the applied policies for afforestation and deforestation. As it turns out, not only do we have a potential switch of regimes for all three controls, but the switches themselves are interdependent.

One can obtain the analytical expressions for the evolution of the state and co-state variables for all possible cases (i.e., before and after the switch). But just as it happened with the problem of the nonforest-owner group, it is not possible to derive the exact switching times analytically.

Denote now by $t_{A}^{c}, t_{D}^{c}$ and $t_{V}^{c}$ the switching time for $A, D$ and $V$, respectively. We evaluated the discounted intertemporal sum of joint payoffs for all possible combinations of integer switching times $\left(t_{A}^{c}, t_{D}^{c}, t_{V}^{c}\right)$ using a similar algorithm as before. See Table 2 for a sketch of the algorithm.

The only difference from the previous algorithm is that now the computational complexity is increased as a consequence of the multiplicity of cases. Denote by $\tilde{t}_{A}^{c}, \tilde{t}_{D}^{c}, \tilde{t}_{V}^{c}$ the three integer switching times that yield greater intertemporal payoffs. We computed $\tilde{t}_{A}^{c}, \tilde{t}_{D}^{c}, \tilde{t}_{V}^{c}$ for $T \in\{1,2, \ldots, 100\}$ and for $r \in\{0.01,0.02,0.03\}$. Again, the results are
Table 3

Jointly optimal policies are a function of $T$.

\begin{tabular}{lllll}
\hline Switch & $Z_{1}$ & $Z_{2}$ & $Z_{3}$ & $Z_{4}$ \\
\hline$\tilde{t}_{A}$ & N.A. & $\tilde{t}_{A}=f(T)$ & $T$ & $T$ \\
$\tilde{t}_{D}$ & N.A. & $\tilde{t}_{D}=g(T)$ & $T$ & $T$ \\
$\tilde{t}_{V}$ & N.A. & N.A. & N.A. & $\tilde{t}_{V}=h(T)$ \\
\hline
\end{tabular}

linked to the length of the planning horizon and the discount rate used. We observe that the solutions obtained can be classified into four different groups that coincide with four regions of the parameter space. We denote them by $Z_{1}$ to $Z_{4}$. The boundaries of regions $Z 1-Z 4$ are related to parameter $T$. We denote the limits to these regions by $T_{1}, T_{2}$ and $T_{3}$

The results, which are summarized in Table 3, call for the following comments:

(i) If the problem's planning horizon is short (i.e., $T<T_{1}$ ) we are in region $Z_{1}$ and the cooperative solution coincides with the noncooperative one (i.e., the cooperative solution brings no gain). The label not applicable (N.A.) is used here to denote that there is no switching time and that the solution coincides with the status quo.

(ii) If we are in region $Z_{2}$ (i.e., $T_{1} \leq T<T_{2}$ ), then it is jointly optimal to afforest at the maximum rate for some time and then to switch to afforestation $A_{\text {min }}$ at some time before the end of the planning horizon. It is not optimal to afforest all the time and we have that $A^{c}=A_{\max }$ if $t<\tilde{t}_{A}^{c}$ and $A^{c}=A_{\min }$ if $t \geq \tilde{t}_{A}^{c}$. We use the notation $\tilde{t}_{A}^{c}=f(T)$ to denote the fact that the switching time depends on $T$. In fact $f(T)$ is an increasing function of $T$. Clearly, for larger values of $T$, it is optimal to switch later. The same reasoning applies for $\tilde{t}_{D}^{c}$. In this case, though, we have $D^{*}=D_{\min }$ if $t<\tilde{t}_{D}^{c}$ and $D^{*}=D_{\max }$ if $t \geq \tilde{t}_{D}^{c}$.

(iii) If we are in region $Z_{3}$ (i.e., $T_{2} \leq T<T_{3}$ ), then it is optimal to apply $A^{*}=A_{\max }$ and $D^{*}=D_{\min }$ all along. We have used the notation $\tilde{t}_{A}^{c}=\tilde{t}_{D}=T$ to differentiate it from label N.A. Recall that label N.A. was used to denote that there is no switch and that the optimal policy is identical to the status quo one (i.e., $A^{c}=$ $A_{\text {min }}$ and $\left.D^{c}=D_{\text {max }} \forall t \in 0, T\right]$ ) whereas in region $Z_{3}$ we also have that there is no switch, but the optimal policy is to apply $A^{c}=A_{\max }$ and $D^{c}=D_{\min }$ throughout.

(iv) Finally, region $Z_{4}$ is identical to region $Z_{3}$ except for the emissions policy. If $T \geq T_{3}$ then it is certain that we will have a jump from $V_{\max }$ to $V_{\min }$ at some point in time $\tilde{t}_{V}^{c}$. The time of the switch is also a function of $T$.

The impact of cooperation is more intense and the solution is more environmentally friendly as we move from region $Z_{1}$ (no gain from cooperation) to region $Z_{4}$. When the discount rate is smaller, the environmental damage is further internalized. Table 4 shows the values of $T_{1}$ to $T_{3}$ for different values in the discount rates. It is not surprising that when the discount rates are smaller, the threshold planning horizons $\left(T_{1}, T_{2}, T_{3}\right)$ between regions $Z_{1}, Z_{2}, Z_{3}$ and $Z_{4}$ are shifted downwards (see Table 4). ${ }^{14}$

Appendix F shows that these results seem quite robust to changes in the environmental damage parameter (parameter $c$ ).

\footnotetext{
${ }^{14}$ We recall that the planning horizon is not a control variable, but a parameter that reflects how forward looking the society is. In this sense, it is not clear what it takes to shift from $Z_{1}$ to $Z_{4}$. What can be conjectured is that as societies become richer and (i) politicians become increasingly aware of the importance to design long-term and sound environmental policies and (ii) voters-consumers become more aware of the long-term consequences of their actions today, then it will be more likely to adopt policies that lead to using the planning horizon that will help in shifting toward region $Z_{4}$.
} 
Table 4

Threshold times are a function of the discount rate.

\begin{tabular}{llll}
\hline Discount & $T_{1}$ & $T_{2}$ & $T_{3}$ \\
\hline$r=1 \%$ & 11 & 19 & 36 \\
$r=2 \%$ & 12 & 20 & 38 \\
$r=3 \%$ & 12 & 21 & 41 \\
\hline
\end{tabular}

\section{Sharing the Gain of Cooperation}

In this section, we determine a time-consistent allocation of the dividend of cooperation among the two players. We have shown that joint payoffs are greater in the cooperative setting provided that $T \geq T_{1}$. This is due to the damage reduction generated by increased afforestation effort and lower deforestation rates. Cooperation, however, does not bring gains to both players. The non-forestowner group gains from the lower environmental damage, while forest owners lose by applying forest policies that are environmentally friendly but revenue harming.

Let $\left(x_{\tau}, \tau\right)$ be the position of the game at time $\tau \in[0, T]$ and the state-vector value $x_{\tau}$. Denoted by $J_{i}^{c}\left(x_{0}, t_{0}\right)$ the payoff-to-go that player $i \in\{\mathcal{O}, \ominus\}$ obtains if the game is played cooperatively throughout the planning horizon, and by $J_{i}^{n c}\left(x_{0}, t_{0}\right)$ its non-cooperative counterpart. The difference $J_{\ominus}^{c}\left(x_{0}, t_{0}\right)-J_{\ominus}^{n c}\left(x_{0}, t_{0}\right)$ measures the individual gain that non-owners obtain from cooperation. By the same token $J_{\mathcal{O}}^{\text {nc }}$ $\left(x_{0}, t_{0}\right)-J_{\mathcal{O}}^{c}\left(x_{0}, t_{0}\right)$ represents the loss that forest owners have in the cooperative setting vis-à-vis the non-cooperative one. These two quantities are a function of $T$ and $r$. We compare the cooperative gains of non-owners and the cooperative losses of forest owners in Fig. 5 for $r=2 \%$.

The cooperative gain by the non-forest-owner group is represented by the solid line, and the loss by forest owners by the dashed one. The vertical difference between these two lines measures the dividend of cooperation given by

$D C=\left[J_{\ominus}^{c}\left(x_{0}, t_{0}\right)+J_{\mathcal{O}}^{c}\left(x_{0}, t_{0}\right)\right]-\left[J_{\ominus}^{n c}\left(x_{0}, t_{0}\right)+J_{\mathcal{O}}^{n c}\left(x_{0}, t_{0}\right)\right]$,

for any given planning horizon $T$. We obtain empirically that $D C>0$ for $T \geq T_{1}$ (with $T_{1}=11$ years), and $D C=0$, otherwise. This means that, unless the planning horizon is longer than $T_{1}$ (which is 11 years for $r=$ $2 \%$, cooperation is useless. For intermediate values of $T$, i.e., $T_{1} \leq T<T_{3}$, we have $D C>0$, and it is optimal to mitigate future damages by increasing afforestation and decreasing deforestation, but not to abate emissions. If the planning horizon is sufficiently long, that is, $T \geq T_{3}$, then it is optimal both to mitigate (from the beginning) and to abate emissions (from time $\tilde{t}_{V}^{c}$ onwards). As emissions abatement has a greater cost than increasing afforestation or decreasing deforestation, it is preferable to start by applying less costly measures first and then move into more costly ones as environmental damages increase.

Although the total dividend of cooperation is by virtue of joint optimization always non-negative, this does not mean that cooperation is Pareto improving. In our case, it is clear that for cooperation to be implemented, the non-owners need to compensate forest owners for their losses. There are many solution concepts ${ }^{15}$ in cooperative games that address the problem of sharing $D C$. We adopt here the often used Nash-bargaining procedure, which gives a unique, fair and Paretoimproving solution. ${ }^{16}$ The Nash-bargaining solution (NBS) allocates to

\footnotetext{
15 For example, the Kalai and Smorodinsky (1975) bargaining solution or the Kalai (1977) egalitarian principle.

${ }^{16}$ The unfamiliar reader with the Nash-bargaining solution can consult, e.g., Wikipedia (http://en.wikipedia.org/wiki/Bargaining_problem) for a quick introduction.
}

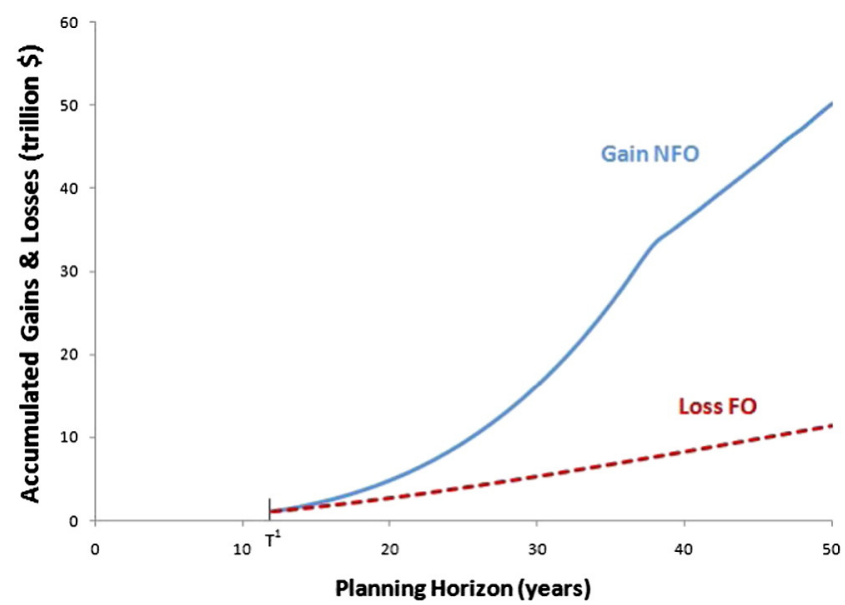

Fig. 5. Cooperation gains and losses by NFO and FO.

each player his non-cooperative outcome plus half of the dividend of cooperation, that is,

$J_{i}^{N B S}\left(x_{0}, t_{0}\right)=J_{i}^{n c}\left(x_{0}, t_{0}\right)+\frac{1}{2} \sum_{i \in\{\mathcal{O}, \ominus\}}\left(J_{i}^{c}\left(x_{0}, t_{0}\right)-J_{i}^{n c}\left(x_{0}, t_{0}\right)\right)$.

\subsection{Time-consistent Sharing Schedule}

Although the Nash-bargaining outcomes defined in Eq. (19) are Pareto-improving with respect to non-cooperative outcomes, it does not guarantee that the players will indeed continue to implement over time their part of the cooperative solution. In fact, the agreement will not be sustained if it is optimal, for at least one of them, to deviate to a non-cooperative mode of play at an intermediate date $\tau \in(0, T]$. This would mean that the agreement designed at the initial date for the whole duration of the game is not time consistent. (For a tutorial on time consistency in differential games, the reader may consult Yeung and Petrosjan (2005) or Zaccour (2008).) Formally, we say that a cooperative solution (here NBS) is time consistent at $\left(x_{0}, t_{0}\right)$ if, at any position $\left(x_{\tau}^{*}, \tau\right)$, and for all $\tau \in\left[t_{0}, T\right]$, it holds that

$J_{i}^{N B S}\left(x_{\tau}^{*}, \tau\right) \geq J_{i}^{n c}\left(x_{\tau}^{*}, \tau\right), \quad i \in\{\mathcal{O}, \ominus\}$,

where $x^{*}$ denotes the cooperative state trajectory. Note that the comparison of payoffs-to-go in Eq. (20) at any $\tau \in\left[t_{0}, T\right]$ is carried out along the cooperative state trajectory, that is, under the assumption that the players have cooperated until $\tau$.

Solving the time-consistency problem amounts to finding payment functions $\omega_{i}(t), i \in\{\mathcal{O}, \ominus\}, t \in\left[t_{0}, T\right]$, such that the following two properties hold:

Full allocation : $\int_{t_{0}}^{T} e^{-r t} \omega_{i}(t) d t=J_{i}^{N B S}\left(x_{0}, t_{0}\right)$,

Time consistency : $J_{i}^{N B S}\left(x_{0}, t_{0}\right)=\int_{t_{0}}^{\tau} e^{-r t} \omega_{i}(t) d t+e^{-r \tau} J_{i}^{N B S}\left(x_{\tau}^{*}, \tau\right)$.

The first property states that the total payments that each player receives overtime must correspond to what he is entitled to, as determined by the Nash-bargaining solution. To interpret the second condition, assume that the players wish to renegotiate the initial agreement at (any) intermediate instant of time $\tau$. At this moment, the position of the game is $\left(x_{\tau}^{*}, \tau\right)$, meaning that cooperation has prevailed from the initial time until $\tau$, and that each player $i$ would have been allocated a stream of monetary amounts given by the first right-hand-side term. Now, if the subgame starting with initial condition $x(\tau)=x_{\tau}^{*}$, is played 
cooperatively, then player $i$ will get his NBS-value component in this game given by the second right-hand-side term of Eq. (22). If what he has been allocated until $\tau$ and what he will be allocated from this date onward add up to his payoff in the original agreement, i.e., his NBS value $J_{i}^{N B S}\left(x_{0}, t_{0}\right)$, then a renegotiation would leave the original agreement unaltered. If one can find a vector $\omega(t)=\left(\omega_{\mathcal{O}}(t), \omega_{\ominus}(t)\right)$ such that Eq. (22) holds true, then the allocation over time $\omega(t)$ is time consistent. To obtain the value $\omega_{i}(t), t \in\left[t_{0}, T\right]$, it suffices to differentiate Eq. (22), that is,

$\omega_{i}(\tau)=r J_{i}^{N B S}\left(x_{\tau}^{*}, \tau\right)-\frac{d}{d \tau}\left(J_{i}^{N B S}\left(x_{\tau}^{*}, \tau\right)\right)$

The above formula has a nice interpretation. It allocates to player $i$ at time $\tau$ the interests on cooperative payoff-to-go, minus the variation over time of this payoff-to-go. We have computed forest owners' NBS payoffs-to-go $\left(J_{\mathcal{O}}^{N B S}\left(x_{\tau}^{*}, \tau\right)\right)$ and their non-cooperative payoffs-togo $\left(J_{\mathcal{O}}^{n c}\left(x_{\tau}^{*}, \tau\right)\right)$. The results are plotted in Fig. 6 .

In Fig. 6 we see that the NBS outcomes (solid line) dominate their non-cooperative deviation counterparts (dotted line) for any time $\tau$. This means that cooperation is time consistent and non-forest owners have no incentive to deviate from the agreement. The vertical difference between these two curves illustrates, at each point in time, the amount of money that forest owners gain by cooperating (i.e., their net cooperation gain-to-go). In particular, for $t=0$ we obtain the net discounted gain at the beginning of the planning horizon. Fig. 6 also displays forest owners' sum of discounted cooperative payoffs-to-go before transfers are applied (dashed line), $J_{\mathcal{O}}^{c}\left(x_{\tau}^{*}, \tau\right)$. The vertical difference between $J_{\mathcal{O}}^{N B S}$ $\left(x_{\tau}^{*}, \tau\right)$ and $J_{\mathcal{O}}^{c}\left(x_{\tau}^{*}, \tau\right)$ gives the value of the compensation payments-togo. Recall that, by cooperating, forest owners reduce their deforestation and therefore suffer economic losses. The amount of money with which they are compensated has to more than cover this loss. For a more straightforward visualization of the intertemporal decomposition of these NBS compensation payments we present Fig. 7.

Fig. 7 shows the yearly decomposition of the economic transfers. These transfers, which have been discounted, are decreasing in time. The main reason why less money is required to bind forest owners into the agreement as time goes by is simply that the gain that forest owners can make by deviating from it is decreasing in time. Therefore, a lower amount is necessary. The economic interpretation is that the compensating mechanism based on the Nash-bargaining solution is time consistent, that is, cooperation is implementable and sustainable overtime.

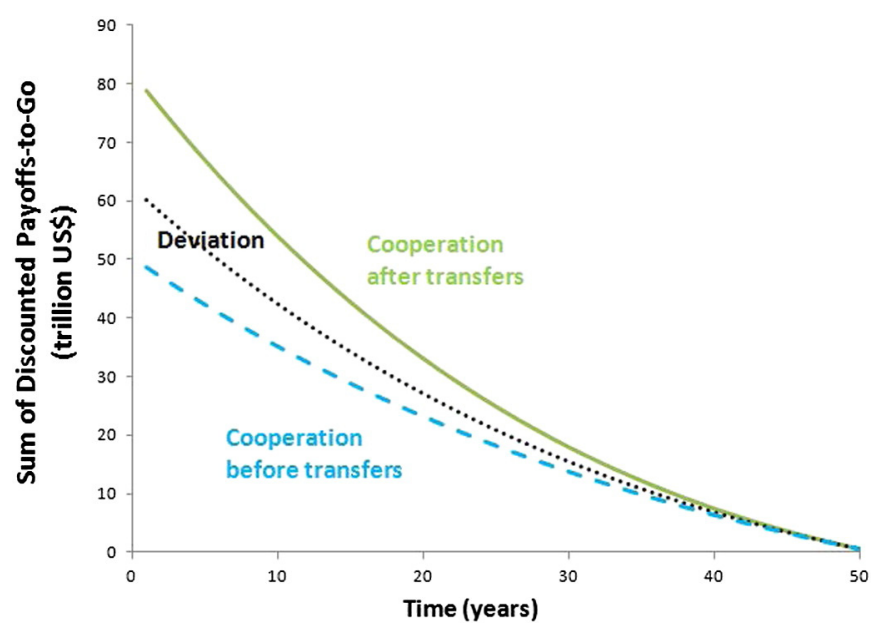

Fig. 6. Time consistency: non-forest-owner group.

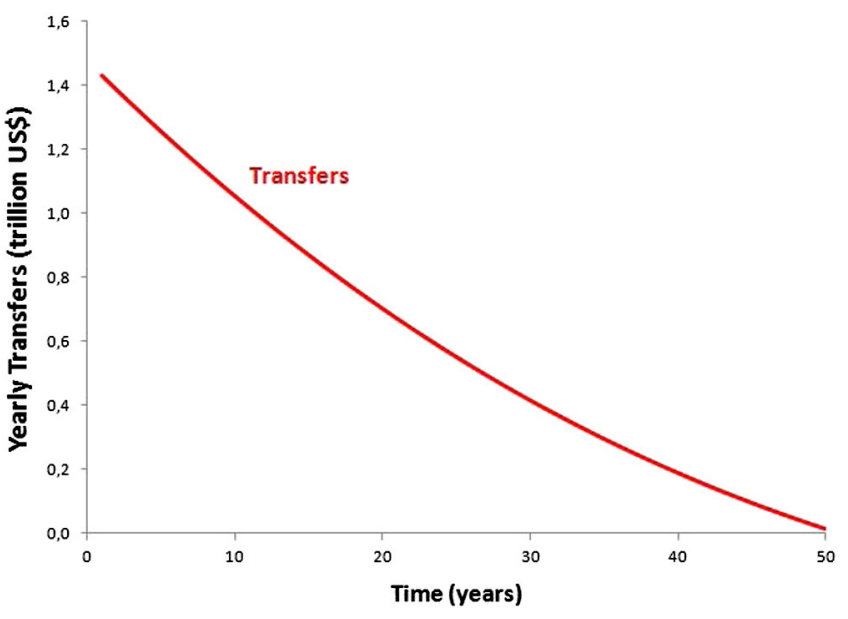

Fig. 7. NBS compensation transfers discounted per unit of time.

\section{Conclusions}

In this paper we propose a model with two types of players: forest owners and the non-forest-owner group, where the first group is interested in maximizing economic revenues and the second group is concerned with the conservation of the forest. A number of papers in the dynamic game literature (e.g. van Soest and Lensink (2000), Fredj et al. (2004, 2006), Martín-Herrán and Tidball (2005) and MartínHerrán et al. (2006)) have looked at the conditions necessary to build environmental agreements that are both credible and sustainable when it comes to stopping deforestation. In these papers the environmentally aware players (non-forest-owners) are willing to compensate forest owners in order to have deforestation reduced.

We look at the same problem from a different angle: forests play an important role in mitigating climate change through carbon sequestration. Deforestation has great impact not just in forest depletion but also on the evolution of the atmospheric stock of greenhouse gases.

There exist a number of papers in the dynamic-game literature that consider environmental damages from emissions in a dynamic setting: for example, the early papers by van der Ploeg and de Zeeuw (1992), Long (1992), Dockner and Long (1993), and the literature review by Jørgensen et al. (2010), and recently, Masoudi and Zaccour (2013). In this literature, emissions are a control variable and the issue is to determine the optimal emissions rate so as to reduce the environmental damage coming from the excessive accumulation of GHGs. Typically, these models concentrate on the difficulty of coordinating optimal emission levels and treat carbon sequestration as exogenously given. We model the two issues together and account explicitly for the role of forests as a carbon sink and treat carbon sequestration not as exogenously given, but rather as the consequence of endogenous decisions, much in the same way as in Andrés-Domenech et al. (2011) except that here we are not in a pure control setting but there exists interaction among the players themselves.

In our proposal, forest owners have an incentive to deforest to increase their economic revenues, while non-owners suffer a negative externality from deforestation due to the so-called reduced-carbonsequestration effect, which states that a tree that is cut cannot grow and hence cannot sequester carbon. We model the economic incentives of both types of players and explore the conditions that make environmental cooperation strictly welfare improving. Cooperation brings greener outcomes and makes it possible to partly internalize the positive externality created by carbon sequestration by forests.

Three different mechanisms to reduce GHG accumulation are proposed: abatement of emissions, increases in afforestation, and decreases in deforestation. The results show that when the perceived 
environmental damage is small (i.e., short planning horizons and/or high intertemporal discount rates), cooperation brings little or no gain. However, as the environmental damages increase, it becomes jointly optimal to have some afforestation effort and deforestation reduction. If the environmental damages coming from the excessive accumulation of greenhouse gases are sufficiently high, then it will be optimal to combine forestation efforts (reduced deforestation and increased afforestation) with emissions abatement. Reducing emissions is more expensive but also more effective in mitigating future environmental damage coming from the excessive accumulation of GHGs.

Our results convey a doubly positive message: first, considering forests' carbon-sequestration potential can make a significant difference toward stopping the destruction of the forests. Second, international cooperation can bring sound economic and environmental gains. Cooperation however will not arise spontaneously. For cooperation to exist, some sort of intertemporal compensating transfer mechanism is needed. It is important to design this transfer mechanism correctly; otherwise the agents may have an economic incentive to withdraw from it, which in turn, will lead to worse environmental outcomes. In order for an environmental agreement to be credible, time consistency is required, i.e., it has to be economically optimal for all the players involved to comply with the agreement at all times. We show that applying an intertemporal decomposition of the Nash bargaining scheme allows us to obtain time consistent outcomes.

The results obtained are very promising and can be applied to design time-consistent intertemporal payments in REDD and REDD + agreements. For instance, the dichotomy used here (owners and non-owners) can be easily extended to consider countries (e.g., developing countries with forests on the one hand, and developed countries willing to pay for forest conservation on the other). Also, we have modeled here the payment of one ecosystem service (carbon), the framework and methodology used lend themselves to modeling the payment for other ecosystem services such as water provision, biodiversity, land protection, etc. Since forest conservation is complementary with the provision of most of these services, by including more services in our model, the range of parameters (time horizon and discount rates) for which cooperation through reduced deforestation is overall welfare improving will increase. This in turn will imply that both the economic benefits arising from cooperation and the amount of money to be shared will increase the greater the amount of services considered.

The conclusions arising from our work suggest that compensation schemes based on services of the same type should not be constant, but rather a decreasing function of time with greater compensation payments allocated in the short run. This is the only way in which payments can credibly bind forest owners to cooperate. Some aspects such as the degree of economic development of (forestry) countries have not been explicitly modeled here. Including such aspects could only reinforce the strength of our conclusions (i.e., that transfers have to be greater in the short run, and then decrease as time goes by) since the opportunity cost to reduce deforestation is greater in a developing country where the average revenue is low; see e.g., Angelsen and Rudel (2013) and Wolfersberger et al (2014).

All that being said, there are many aspects that were not considered and that call for a critical interpretation of the results: carbon sequestration by the oceans may be affected by the excessive acidification of seawater which has not been specifically modeled here. Also a more comprehensive dynamics of the accumulation of greenhouse gases should consider emissions related to land-use change. More thorough research should integrate these aspects, with yet more pessimistic conclusions expected.

\section{Acknowledgments}

We thank three anonymous reviewers and the Editor for their helpful comments. The second author's research is partially supported by MEC under projects ECO2011-24352 and ECO201452343-P co-financed by FEDER funds and the COST Action IS1104 "The EU in the new economic complex geography: models, tools and policy evaluation". The third author's research is supported by NSERC, Canada.

\section{Appendix A. Supplementary Data}

Supplementary data to this article can be found online at http://dx. doi.org/10.1016/j.ecolecon.2015.06.016.

\section{References}

Andrés-Domenech, P., Saint-Pierre, P., Zaccour, G., 2011. Forest conservation and $\mathrm{CO}_{2}$ emissions: a viable approach. Environ. Model. Assess. 16 (6), 519-539.

Angelsen, A., Rudel, T.K., 2013. Designing and implementing effective REDD + policies: a 575 forest transition approach. Rev. Environ. Econ. Policy 7 (1), 91-113.

Bahn, O., Haurie, A., Malhamé, R., 2008. A stochastic control model for optimal timing of climate policies. Automatica 44, 1545-1558.

Barbier, E.B., Burgess, J.C., 2001. The economics of tropical deforestation. J. Econ. Surv. 15 (3), 413-433.

Barbier, E.B., Rauscher, M., 1994. Trade, tropical deforestation and policy intervention. Environ. Resour. Econ. 4 (1), 75-90.

Benchekroun, H., Long, N.V., 2002. On the multiplicity of efficiency-inducing tax rules. Econ. Lett. 76, 331-336.

Breton, M., Zaccour, G., Zahaf, M., 2005. A differential game of joint implementation of environmental projects. Automatica 41, 1737-1749.

Breton, M., Martín-Herrán, G., Zaccour, G., 2006. Equilibrium investment strategies in foreign environmental projects. J. Optim. Theory Appl. 130 (1), 23-40.

Dockner, E.J., Long, N.V., 1993. International pollution control: cooperative versus noncooperative strategies. J. Environ. Econ. Manag. 24, 13-29.

Food and Agriculture Organization of the United Nations (FAO), 2006. Global Forest Resources Assessment 2005.

Food and Agriculture Organization of the United Nations (FAO), 2010. Global Forest Resources Assessment 2010.

Fredj, K., Martín-Herrán, G., Zaccour, G., 2004. Slowing deforestation rate through subsidies: a differential game. Automatica 40 (2), 301-309.

Fredj, K., Martín-Herrán, G., Zaccour, G., 2006. Incentive mechanisms to enforce sustainable forest exploitation. Environ. Model. Assess. 11 (2), 145-156.

Intergovernmental Panel on Climate Change (IPCC), 2000. Land use, land-use change and forestry. Special Report. Cambridge University Press, Cambridge.

Intergovernmental Panel on Climate Change (IPCC), 2007. Climate Change 2007: Impacts, Adaptation and Vulnerability, Fourth Assessment Report. Cambridge University Press, Cambridge.

Jørgensen, S., Martín-Herrán, G., Zaccour, G., 2010. Dynamic games in the economics and management of pollution. Environ. Model. Assess. 15 (6), 433-467.

Kalai, E., 1977. Proportional solutions to bargaining situations: intertemporal utility comparisons. Econometrica 45 (7), 1623-1630.

Kalai, E., Smorodinsky, M., 1975. Other solutions to Nash's bargaining problem. Econometrica 43 (3), 513-518.

Le Quéré, C. Raupach, M.R. Canadell, J.G., Marland, G, et al., 2009. Trends in the sources and sinks of carbon dioxide. Nat. Geosci. 2, 831-836.

Long, N.V., 1992. Pollution control: a differential game approach. Ann. Oper. Res. 37, 283-296.

Martín-Herrán, G., Tidball, M., 2005. Transfer mechanisms inducing a sustainable forest exploitation. In: Deissenberg, C., Hartl, R. (Eds.), Optimal Control and Dynamic Games: Applications in FinanceManagement Science and Economics. Springer, The Netherlands, pp. 85-103.

Martín-Herrán, G., Cartigny, P., Motte, E., Tidball, M., 2006. Deforestation and foreign transfers: a Stackelberg differential game approach. Comput. Oper. Res. 33 (2) 386-400.

Masoudi, N., Zaccour, G., 2013. A differential game of international pollution control with evolving environmental costs. Environ. Dev. Econ. 18, 680-700.

Van der Ploeg, F., De Zeeuw, A., 1992. International aspects of pollution control. Environ Resour. Econ. 2 (2), 117-139.

Van Soest, D., Lensink, R., 2000. Foreign transfers and tropical deforestation: what terms of conditionality? Am. J. Agric. Econ. 82 (2), 389-399.

Wolfersberger, J., Amacher, G.S., Delacote, P., Dragicevic, A., 2014. Dynamics of deforestation and reforestation in a developing economy. Working Paper Chaire Economie du Climat.

Yeung, D.W.K., Petrosjan, L.A., 2005. Cooperative Stochastic Differential Games. Springer, New York.

Zaccour, G., 2008. Time consistency in cooperative differential games: a tutorial. INFOR 46 (1), 81-92. 